\title{
Sequence-based typing of genetic targets encoded outside of the O-antigen gene cluster is indicative of Shiga toxin-producing Escherichia coli serogroup lineages
}

\begin{abstract}
Correspondence
Matthew W. Gilmour

Matthew_Gilmour@

phac-aspc.gc.ca
\end{abstract}

Received 7 November 2006

Accepted 28 January 2007

\author{
Matthew W. Gilmour, ${ }^{1,2}$ Adam B. Olson, ${ }^{1}$ Ashleigh K. Andrysiak, ${ }^{2}$ \\ Lai-King $\mathrm{Ng}^{1,2}$ and Linda Chui ${ }^{3}$ \\ ${ }^{1}$ National Microbiology Laboratory, Public Health Agency of Canada, 1015 Arlington Street, \\ Winnipeg, Manitoba R3E 3R2, Canada \\ ${ }^{2}$ Department of Medical Microbiology and Infectious Diseases, University of Manitoba, Winnipeg, \\ Manitoba, Canada \\ ${ }^{3}$ Alberta Provincial Laboratory for Public Health, Edmonton, Alberta, Canada
}

\section{INTRODUCTION}

Shiga toxin-producing Escherichia coli (STEC) are bacterial pathogens that result in both outbreak and sporadic occurrences of human mortality and disease. Symptoms can include bloody and non-bloody diarrhoea, and children are susceptible to renal failure due to haemolytic uraemic syndrome. STEC are transmitted to humans by consumption

Abbreviations: LEE, locus for enterocyte effacement; NM, non-motile; STEC, Shiga toxin-producing Escherichia coli.

The GenBank/EMBL/DDBJ accession numbers for the nucleotide sequences reported in this paper are DQ472524-DQ472651. of contaminated food or water, person-to-person contact or animal-to-person contact, where natural reservoirs include cattle, pigs and sheep (Karch et al., 2005). Serogroup classifications based upon the O-somatic or $\mathrm{H}$-flagellar antigens of STEC provide significant epidemiological information on clinical isolates, and this measure can provide the first indication of relatedness between strains during outbreak investigations. The serogroup is also indicative of the overall genetic relatedness between E. coli strains, including virulence gene content, such as the locus for enterocyte effacement (LEE) pathogenicity island, and the st $x 1$ and stx2 loci encoding Shiga toxins (Prager et al., 2005; Girardeau et al., 2005; Karmali et al., 2003). 
The predominant O-serogroup of STEC that is observed clinically in North America is O157 (Johnson et al., 2006); however, biased sampling likely results from the availability of clinical media and detection reagents that target this serogroup. Directed studies for the isolation and characterization of both $\mathrm{O} 157$ and non-O157 STEC from clinical samples have indicated that the proportion of nonO157 in North America is likely higher than clinical records have indicated (Thompson et al., 2005; Jelacic et al., 2003; Fey et al., 2000). In Canada, over $90 \%$ of STEC strains detected are serotype O157:H7 or O157:nonmotile (NM) (Woodward et al., 2002). The global prevalence of non-O157 includes significant outbreaks of O26, O121, O103, O111 and O145, and in some countries it is recognized that these serogroups exceed the prevalence of O157 STEC (Karch et al., 2005). Furthermore, nonO157 strains have been identified along with O157 strains in clinical samples (Paton et al., 1996), so it is possible that a diagnostic bias towards O157 may prevent the detection of the aetiological STEC serogroup during human illness.

Molecular methods for the characterization and identification of $\mathrm{O}$-antigen determinants have been devised using restriction profiling and allele-specific PCR. The entire Oantigen-encoding gene cluster could be amplified using primers that targeted conserved regions in the neighbouring gnd sequence (encoding 6-phosphogluconate dehydrogenase) and JUMPstart sequence, and enzymic digestion of this amplicon identified RFLPs correlating to O-antigen determinants (Coimbra et al., 2000). This method was problematic due to the length of the amplicon (upwards of $20 \mathrm{kbp}$ ) and the absence of unique restriction profiles for all serotypes. Within the $\mathrm{O}$-antigen gene cluster the $w z x$ and $w z y$ loci encode the $\mathrm{O}$-antigen flippase and polymerase, respectively, and distinct alleles corresponding to each $\mathrm{O}$-serogroup have been used for molecular serogrouping of O103, O157, O26, O113 and O111 strains (Perelle et al., 2005; DebRoy et al., 2004; Paton \& Paton, 1999a; Fratamico et al., 2005; D'Souza et al., 2002). It has been suggested that these assays could replace traditional serological methods (DebRoy et al., 2005); however, the individual tests currently detect only one to three Oserogroups. In the absence of a priori knowledge of a serogroup, a large number of reagents may be required to confirm serogroup identity with these methods. Robust platforms such as DNA microarrays containing $w z x$ and wzy probes targeting up to four E. coli serogroups are currently being investigated (Liu \& Fratamico, 2006), and broad subtyping of STEC has been achieved using allelic variants of a LEE-encoded determinant (Gilmour et al., 2006).

Multilocus sequence typing has been attempted for each of the STEC serotypes O26:H11, O121:H19, O103:H2 or O157:H7, but this method was not appropriate for subtyping because very few polymorphisms were observed between strains of the same serotype (Gilmour et al., 2005; Tarr et al., 2002; Noller et al., 2003; Beutin et al., 2005). The genetic differentiation and subtyping of $E$. coli serotype
O26: H11 was attempted by sequencing 10 loci for 30 strains encoding stxl, or both stx1 and stx2 (Gilmour et al., 2005). Amongst the O26:H11 strains all loci were identical, with the exception of three alleles of $m d h$ and two alleles of $p p k$ that each differed by a single point mutation. Notably, comparative analyses of the $m d h$, gnd, gcl, ppk, metA, ftsZ, relA and met $G$ alleles encoded by O26:H11 STEC cumulatively distinguished this serotype from O157:H7 (Gilmour et al., 2005). The conservation of these loci between $\mathrm{O} 26: \mathrm{H} 11$ strains, and the genetic distance from the other E. coli serotypes suggested that sequence-based typing of additional STEC might reveal serotype-specific alleles. In this study, additional DNA sequence data at these loci was obtained for a range of STEC and a single locus was observed to encode allelic variants correlating to individual STEC O-serogroups. We therefore present a simple molecular method for the identification of STEC serogroups, including both $\mathrm{O} 157$ and non-O157 strains.

\section{METHODS}

Bacterial strains. STEC strains (Table 1) were obtained from the reference stocks of the Enteric Diseases Program at the National Microbiology Laboratory that originated from human sources at various Canadian provincial health laboratories during 1985-2005, or were recent clinical isolates obtained from the Alberta Provincial Laboratory for Public Health (nomenclature XX-YYYY, where XX generally refers to the year of isolation). During the course of these studies, five outbreak-associated STEC isolates were provided by Nova Scotia Public Health, Halifax, Nova Scotia, Canada. Confirmation of $\mathrm{O}: \mathrm{H}$ serotype was completed with antisera prepared at the National Microbiology Laboratory (Ewing, 1986).

PCR and sequencing. Template DNA was prepared by centrifuging $1 \mathrm{ml}$ exponential phase culture grown in brain heart infusion broth, resuspending the pellet in $1 \mathrm{ml}$ TE buffer (Sigma; $10 \mathrm{mM}$ Tris/ $\mathrm{HCl}$, $1 \mathrm{mM}$ EDTA, pH 8.0) and boiling the cells for $15 \mathrm{~min}$. Boiled cells were pelleted, and the supernatant was removed and used as the DNA template in PCR.

Oligonucleotide primers used to amplify segments of mdh, gnd, $g c l$, $p p k$, met $A, f t s Z$, relA and met $G$ are presented in Table 2. PCR was performed with high fidelity Platinum Taq (Invitrogen), following the manufacturer's directions. The thermocycling parameters for $f t s Z$, relA and met $\mathrm{G}$ included an initial denaturation at $94{ }^{\circ} \mathrm{C}$ for $5 \mathrm{~min}$, 35 cycles of denaturation at $94{ }^{\circ} \mathrm{C}$ for $40 \mathrm{~s}$, annealing at $50{ }^{\circ} \mathrm{C}$ for $45 \mathrm{~s}$ and extension at $68{ }^{\circ} \mathrm{C}$ for $45 \mathrm{~s}$, with a final extension at $68{ }^{\circ} \mathrm{C}$ for $5 \mathrm{~min}$. The annealing temperature for $m e t \mathrm{~A}, \mathrm{mdh}, \mathrm{gcl}$ and $p p k$ was $58{ }^{\circ} \mathrm{C}$, and $52{ }^{\circ} \mathrm{C}$ for gnd. PCR products were purified using the QIAquick PCR purification kit (Qiagen) and sequenced using the same primers that generated these amplicons. Sequencing was performed on an ABI3730 (Applied Biosystems) and the data were deposited in GenBank with accession nos DQ472524-DQ472651. Existing genomic sequence data for E. coli O157:H7 EDL933, O157:H7 Sakai, O6:H1 CFT073 and K-12 (GenBank accession nos NC_000913, BA000007, NC_002655, NC_004431) was included in our dataset for each of the above loci. From directed studies against the gnd locus (Tarr et al., 2000; Paton \& Paton, 1999b; Wang et al., 1998), we included sequence data from O157:H7 and O157:NM (GenBank accession nos AF176359, AF176358, AF176357, AF176356, AF176360, AF176361 and AB008676), O113:H2 (AF172324), O111 (AF078736) and non-toxin encoding O157 and O55 (AF176368, AF176367, AF176366, AF176363, AF176362, AF176369 and 
Table 1. Bacterial strains used in this study

Strains characterized during outbreak investigations are identified $(O)$.

\begin{tabular}{|c|c|c|c|c|c|c|c|c|}
\hline Seropathotype ${ }^{\star}$ & Serotype & Strain ID & Source $\dagger$ & $\begin{array}{c}\text { Sequencing } \\
\text { scheme } \neq\end{array}$ & stx 1 & stx2 & LEE $\$$ & Reference \\
\hline \multirow[t]{8}{*}{ A } & $\mathrm{O} 157: \mathrm{H} 7$ & $87-1215$ & NML & 8 loci & + & + & + & Gilmour et al. (2006) \\
\hline & O157: H7 & $01-8110$ & NML & 4 loci & + & + & + & Gilmour et al. (2006) \\
\hline & $\mathrm{O} 157: \mathrm{H} 7$ & 05-0958 & SK HPL & 8 loci & - & + & + & Gilmour et al. (2006) \\
\hline & $\mathrm{O} 157: \mathrm{H} 7$ & 04-4319 & SK HPL & 4 loci & + & - & + & Gilmour et al. (2006) \\
\hline & $\mathrm{O} 157: \mathrm{H7}$ & $03-2641$ & AB PLPH & 4 loci & + & + & + & Gilmour et al. (2006) \\
\hline & $\mathrm{O} 157: \mathrm{NM}$ & $01-6434$ & AB PLPH & 8 loci & + & - & + & Gilmour et al. (2006) \\
\hline & $\mathrm{O} 157: \mathrm{NM}$ & 03-3088 & AB PLPH & 4 loci & + & + & + & This study \\
\hline & $\mathrm{O} 157: \mathrm{NM}$ & $03-5296$ & AB PLPH & 8 loci & + & + & + & Gilmour et al. (2006) \\
\hline \multirow[t]{33}{*}{ B } & $\mathrm{O} 26: \mathrm{H} 11$ & $01-6372$ & NS PHL & 8 loci & + & - & + & Gilmour et al. (2005) \\
\hline & $\mathrm{O} 26: \mathrm{H} 11$ & $03-2816$ & AB PLPH & 8 loci & + & - & + & Gilmour et al. (2005) \\
\hline & $\mathrm{O} 26: \mathrm{H} 11$ & $05-6544$ & NS PHL (O) & gnd & + & - & + & This study \\
\hline & $\mathrm{O} 103: \mathrm{H} 2$ & $99-2076$ & BCCDC & 8 loci & + & - & + & Gilmour et al. (2006) \\
\hline & $\mathrm{O} 103: \mathrm{H} 2$ & $04-2446$ & MB CPL & 8 loci & + & - & + & Gilmour et al. (2006) \\
\hline & $\mathrm{O} 103: \mathrm{H} 2$ & $01-6102$ & SK HPL & 8 loci & + & - & + & Gilmour et al. (2006) \\
\hline & $\mathrm{O} 103: \mathrm{H} 2$ & $03-3967$ & AB PLPH & 4 loci & + & - & + & This study \\
\hline & O103: H11 & 04-3973 & MB CPL & gnd & + & - & + & Thompson et al. (2005) \\
\hline & O103: H11 & $06-4464$ & MB CPL & gnd & + & - & + & This study \\
\hline & $\mathrm{O} 103: \mathrm{H} 25$ & $03-1028$ & MB CPL & gnd & + & - & + & Thompson et al. (2005) \\
\hline & $\mathrm{O} 103: \mathrm{H} 25$ & $03-1030$ & MB CPL & gnd & + & - & + & Thompson et al. (2005) \\
\hline & $\mathrm{O} 103: \mathrm{H} 25$ & 04-3972 & MB CPL & gnd & + & - & + & Thompson et al. (2005) \\
\hline & $\mathrm{O} 103: \mathrm{H} 25$ & $03-2444$ & MB CPL & gnd & + & - & + & Thompson et al. (2005) \\
\hline & $\mathrm{O} 111: \mathrm{NM}$ & 03-3991 & AB PLPH & 4 loci & + & - & + & Gilmour et al. (2006) \\
\hline & $\mathrm{O} 111: \mathrm{NM}$ & 04-3794 & MB CPL & 8 loci & + & + & + & Gilmour et al. (2006) \\
\hline & $\mathrm{O} 111: \mathrm{NM}$ & $98-8338$ & BCCDC & 4 loci & + & - & + & Gilmour et al. (2006) \\
\hline & O111:NM & $00-4748$ & SK HPL & 8 loci & + & + & + & Gilmour et al. (2006) \\
\hline & O111: NM & $00-4440$ & BCCDC & 4 loci & + & - & + & Gilmour et al. (2006) \\
\hline & O111: NM & $01-0252$ & BCCDC & 8 loci & + & + & + & Gilmour et al. (2006) \\
\hline & O111: NM & $01-1215$ & BCCDC & 8 loci & + & - & + & Gilmour et al. (2006) \\
\hline & O121: H19 & $03-2636$ & AB PLPH & 4 loci & - & + & + & Gilmour et al. (2006) \\
\hline & O121: H19 & $03-2642$ & AB PLPH & gnd & - & + & + & Gilmour et al. (2006) \\
\hline & O121: H19 & $03-2832$ & AB PLPH & 8 loci & - & + & + & Gilmour et al. (2006) \\
\hline & O121 : H19 & $05-6541$ & NS PHL (O) & gnd & - & + & + & This study \\
\hline & O121:H19 & $05-6542$ & NS PHL (O) & gnd & - & + & + & This study \\
\hline & O121: H19 & $05-6543$ & NS PHL (O) & gnd & - & + & + & This study \\
\hline & O121:H19 & $00-5288$ & $\mathrm{BCCDC}$ & 8 loci & - & + & + & Gilmour et al. (2006) \\
\hline & $\mathrm{O} 145: \mathrm{NM}$ & $03-4699$ & $\mathrm{AB}$ PLPH & 8 loci & + & - & + & Gilmour et al. (2006) \\
\hline & $\mathrm{O} 145: \mathrm{NM}$ & 04-7099 & MB CPL & gnd & + & - & + & This study \\
\hline & $\mathrm{O} 145: \mathrm{NM}$ & $04-7194$ & MB CPL & gnd & + & - & + & This study \\
\hline & $\mathrm{O} 145: \mathrm{NM}$ & 04-1449 & MB CPL & gnd & + & - & + & This study \\
\hline & $\mathrm{O} 145: \mathrm{NM}$ & $03-6430$ & MB CPL & gnd & + & - & + & Thompson et al. (2005) \\
\hline & $\mathrm{O} 145: \mathrm{NM}$ & $02-5149$ & BCCDC & gnd & + & - & + & This study \\
\hline \multirow[t]{8}{*}{$\mathrm{C}$} & O5: NM & $03-2825$ & AB PLPH & 8 loci & + & - & + & Gilmour et al. (2006) \\
\hline & O5: NM & $03-2682$ & MB CPL & gnd & + & - & + & Thompson et al. (2005) \\
\hline & O91: H21 & $85-489$ & NML & 8 loci & - & + & - & Gilmour et al. (2006) \\
\hline & $\mathrm{O} 113: \mathrm{H} 21$ & 93-0016 & NML & 8 loci & - & + & - & Gilmour et al. (2006) \\
\hline & $\mathrm{O} 113: \mathrm{H} 21$ & $04-1450$ & MB CPL & gnd & - & + & - & Thompson et al. (2005) \\
\hline & O121:NM & $99-4389$ & NML & 8 loci & - & + & + & Gilmour et al. (2006) \\
\hline & $\mathrm{O} 121: \mathrm{NM}$ & $03-4064$ & AB PLPH & 4 loci & - & + & + & This study \\
\hline & $\mathrm{O} 165: \mathrm{H} 25$ & $00-4540$ & BCCDC & 8 loci & - & + & + & Gilmour et al. (2006) \\
\hline \multirow[t]{5}{*}{$\mathrm{D}$} & O6:H34 & $03-5166$ & MB CPL & gnd & - & + & - & Thompson et al. (2005) \\
\hline & $\mathrm{O} 45: \mathrm{H} 2$ & $05-6545$ & NS PHL & gnd & + & - & + & This study \\
\hline & $\mathrm{O} 45: \mathrm{H} 2$ & $04-2445$ & MB CPL & gnd & + & - & + & Thompson et al. (2005) \\
\hline & $\mathrm{O} 55: \mathrm{H} 7$ & 05-0376 & NML & gnd & + & - & + & This study \\
\hline & $\mathrm{O} 85: \mathrm{H} 1$ & 03-3638 & AB PLPH & 4 loci & - & + & - & This study \\
\hline
\end{tabular}


Table 1. cont.

\begin{tabular}{|c|c|c|c|c|c|c|c|c|}
\hline Seropathotype ${ }^{*}$ & Serotype & Strain ID & Source $\dagger$ & $\begin{array}{c}\text { Sequencing } \\
\text { scheme } \neq\end{array}$ & stx 1 & stx 2 & LEE $\$$ & Reference \\
\hline \multirow{14}{*}{ NA } & O115:H18 & 03-3645 & AB PLPH & 4 loci & + & + & - & This study \\
\hline & $\mathrm{O} 117: \mathrm{H} 7$ & 02-0035 & BCCDC & gnd & + & - & - & This study \\
\hline & $\mathrm{O} 117: \mathrm{H7}$ & $02-4495$ & BCCDC & gnd & + & + & - & This study \\
\hline & $\mathrm{O} 146: \mathrm{H} 21$ & $02-7808$ & BCCDC & gnd & + & - & - & This study \\
\hline & O177: NM & 03-3974 & AB PLPH & 4 loci & - & + & + & This study \\
\hline & $\mathrm{O} 177: \mathrm{NM}$ & 06-5121 & NS PHL (O) & gnd & - & + & + & This study \\
\hline & $\mathrm{O} 1: \mathrm{H} 7$ & 03-3964 & AB PLPH & 4 loci & - & - & - & This study \\
\hline & $\mathrm{O} 2: \mathrm{H} 4$ & 03-2815 & AB PLPH & 4 loci & - & - & - & This study \\
\hline & $\mathrm{O} 4: \mathrm{H} 5$ & 03-3266 & AB PLPH & 4 loci & - & - & - & This study \\
\hline & O6:H1 & $03-2638$ & AB PLPH & 4 loci & - & - & - & This study \\
\hline & O51:NM & $04-2640$ & MB CPL & gnd & - & - & - & This study \\
\hline & O91: H10 & $03-3269$ & AB PLPH & 4 loci & - & - & - & This study \\
\hline & O98:NM & $02-7464$ & NB PHL & gnd & - & - & - & This study \\
\hline & O117: H25 & $02-0714$ & NB PHL & gnd & - & - & - & This study \\
\hline
\end{tabular}

${ }^{*} \mathrm{NA}$, Not applicable. Strains that do encode stx are not classified in the seropathotype scheme (Karmali et al., 2003).

$\dagger$ AB PLPH, Alberta Provincial Laboratory for Public Health; BCCDC, British Columbia Centre for Disease Control; MB CPL, Manitoba Cadham Provincial Laboratory; NML, National Microbiology Laboratory standard strain; NB PHL, New Brunswick Public Health Laboratory; NS PHL, Nova Scotia Public Health Laboratory; SK HPL, Saskatchewan Health Provincial Laboratory.

¥DNA sequencing was performed for 8 loci ( $m d h, g n d, g c l, p p k$, metA, ftsZ, relA and met $G$ ), 4 loci ( $g n d, g c l$, ppk and relA) or solely the $g n d$ locus. $\S$ As determined by PCR screening for the espZ gene (Gilmour et al., 2006).

AF176373). Our previously acquired sequence data from $\mathrm{O} 26: \mathrm{H} 11$, $\mathrm{O} 26: \mathrm{H} 6$ and O26:H32 strains were also included (GenBank accession nos AY973395-AY973421; Gilmour et al., 2005).

Bioinformatics. Multiple sequence alignments were completed using ClustalW (www.ebi.ac.uk/clustalw/), neighbour-joining trees were constructed with Hasegawa-Kishino-Yano (HKY85) distance correction using SplitsTree4 (Huson, 1998), and genetic diversity statistics were calculated using DnaSP 4.10 .3 (Rozas et al., 2003). Pairwise global alignments were calculated using Align (www.ebi.ac.uk/ emboss/align/\#).

\section{RESULTS AND DISCUSSION}

\section{Sequence typing correlates to 0 -antigen serogroups}

The alleles of $m d h, g n d, g c l, p p k, m e t A, f t s Z$, relA and met $G$ encoded by O26:H11 STEC cumulatively distinguished this serotype from O157:H7 (Gilmour et al., 2005), and the corresponding segments of these loci were sequenced for STEC serotypes O111:NM, O113:H21, O157:NM, $\mathrm{O} 145: \mathrm{NM}, \mathrm{O} 91: \mathrm{H} 21, \mathrm{O} 121: \mathrm{H} 19, \mathrm{O} 121: \mathrm{NM}, \mathrm{O} 103: \mathrm{H} 2$, O165:H25 and O5:NM. This panel of STEC strains included isolates from each of the most predominant $\mathrm{O}$ serogroups and $\mathrm{O}: \mathrm{H}$-serotypes observed in Canada (Gilmour et al., 2005, 2006), and amongst individual serotypes, strains with different stx genotypes were included when available (Table 1). This sequence dataset was compared to previously published sequence data for STEC serotypes O157:H7 and O26:H11, as well as nontoxin producing $\mathrm{O} 26: \mathrm{H} 32, \mathrm{O} 26: \mathrm{H} 6, \mathrm{~K} 12$ and $\mathrm{O} 6: \mathrm{H} 1$ (strain CFT073) strains using the 4464 nucleotide concatenate of the eight genetic determinants (Fig. 1). Each of the examined serogroups had distinct sequence types, including NM STEC strains of O121 and O157, were 99.8 and $99.9 \%$ identical to $\mathrm{O} 121: \mathrm{H} 19$ and $\mathrm{O} 157: \mathrm{H} 7$ strains, respectively. The observed phylogenetic separation between serogroups, and homogeneity within strains of the same serogroup, indicated that these genetic traits have been acquired by and vertically inherited within individual STEC serogroup lineages.

\section{Molecular-based serogrouping with four loci}

Additional sequencing was performed at selected loci in an expanded panel of strains to determine if the phylogenetic separation observed between serogroups was maintained in a larger dataset (Table 1). The genetic determinants that contributed the majority of the observed genetic diversity (gnd and $g c l$; Table 3 ) or encoded putative serogroupspecific regions ( $p p k$ and $r e l A$; data not shown) were selected for further study. This panel included further 
Table 2. Oligonucleotides used in this study

\begin{tabular}{|c|c|c|c|c|}
\hline Oligonucleotide & Target & Sequence $\left(5^{\prime}\right.$ to $\left.3^{\prime}\right)$ & Product size (bp) & Reference \\
\hline GIL213 & $f t s Z$ & GATCACTGAACTGTCCAAGCATG & 450 & Gilmour et al. (2005) \\
\hline GIL214 & $f t s Z$ & TCAAGAGAAGTACCGATAACCAC & & \\
\hline gcl-F & $g c l$ & GCGTTCTGGTCGTCCGGGTCC & 758 & Adiri et al. (2003) \\
\hline gcl-R & $g c l$ & GCCGCAGCGATTTGTGACAGACC & & \\
\hline gnd-F & gnd & GGCTTTAACTTCATCGGTAC & 712 & Noller et al. (2003) \\
\hline gnd-R & gnd & TCGCCGTAGTTCAGATCCCA & & \\
\hline mdh-F & $m d h$ & CAACTGCCTTCAGGTTCAGAA & 580 & Noller et al. (2003) \\
\hline mdh-R & $m d h$ & GCGTTCTGGATGCGTTTGGT & & \\
\hline metA-F & metA & CGCAACACGCCCGCAGAGC & 601 & Adiri et al. (2003) \\
\hline metA-R & metA & GCCAGCTCGCTCGCGGTGTATT & & \\
\hline GIL219 & $m e t G$ & TGGCTGACCCGCAGTTGTAC & 503 & Gilmour et al. (2005) \\
\hline GIL220 & $m e t G$ & GGTCAACTTTGGCGAAGTCGTC & & \\
\hline ppk-F & $p p k$ & TGCCGCGCTTTGTGAATTTACCG & 758 & Adiri et al. (2003) \\
\hline ppk-R & $p p k$ & CCCCGGCGCAGAGAAGATAACGT & & \\
\hline GIL215 & relA & TCTGTTTCCTCCGAACAGGTCG & 470 & Gilmour et al. (2005) \\
\hline GIL216 & relA & ACAATACGTACCGCACGCACATC & & \\
\hline
\end{tabular}

strains from the serotypes represented in Fig. 1, as well as seropathotype $\mathrm{D}$ and non-toxin encoding $E$. coli strains recovered from paediatric stool samples (L. Chui, unpublished data). The overall genetic distinction between STEC serogroups (as determined in the eight locus scheme) was also represented amongst these four loci, and the additional strains and serogroups (Fig. 2).

\section{Molecular-based serogrouping with the gnd locus}

The gnd locus was the most genetically diverse of all examined loci (Table 3), and notably, this determinant is immediately adjacent to the O-antigen gene cluster. Additional sequencing of the 643 bp region of gnd was performed (Table 1), and gnd sequence data available in
GenBank for O157, O113 and O111 STEC, as well as nontoxin encoding $\mathrm{O} 157$ and O55 strains, were also included in comparative analyses. In total, gnd DNA sequences were collected from 144 strains and 26 O-serogroups (comprising $42 \mathrm{O}: \mathrm{H}$-serotypes). The overall genetic distinction between serogroups (as determined in the eight and four loci schemes) was also represented in this single locus, as each examined STEC O-serogroup encoded a unique gnd allele (Fig. 3). For some of the most clinically significant STEC serogroups (O157, O26, O121, O145, O111 and O103) the gnd DNA sequences were compared between multiple strains (from 5 to 43 sequences), and for each serogroup all STEC strains encoded an identical gnd allele (Fig. 3). The only exception was O157:H7 strain 87-16 (GenBank accession no. AF176360), which encoded a

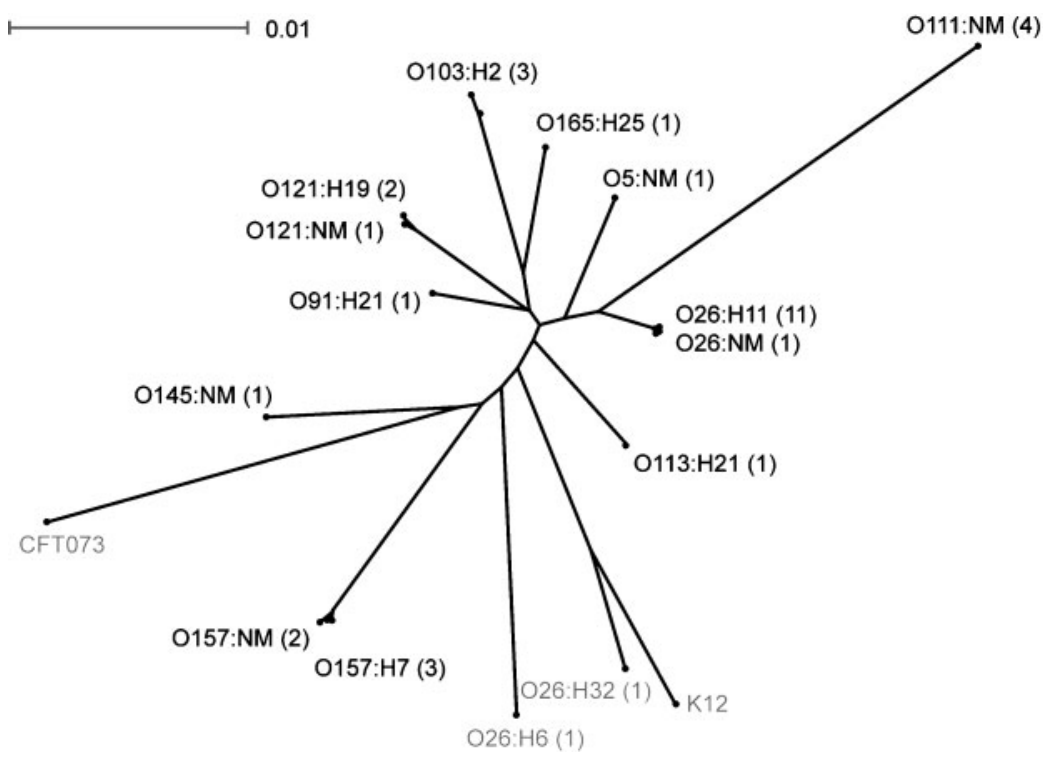

Fig. 1. Phylogeny of the concatenated segments of $m d h$, gnd, $g c l, p p k, m e t A, f t s Z, r e l A$ and $\operatorname{met} G$ encoded by $E$. coli. This is based upon a neighbour-joining tree constructed with Hasegawa-Kishino-Yano (HKY85) distance correction. Sequences obtained from GenBank are identified in Methods. The serotype of strain $\mathrm{K}-12$ was not designated, and the serotype of uropathogenic strain CFT073 was O6:K2:H1. Shiga toxin-producing serotypes are indicated in black type, and strains not encoding stx are indicated in grey. The number of sequences per serotype is indicated in parentheses. Bar, scale of the distance score. 


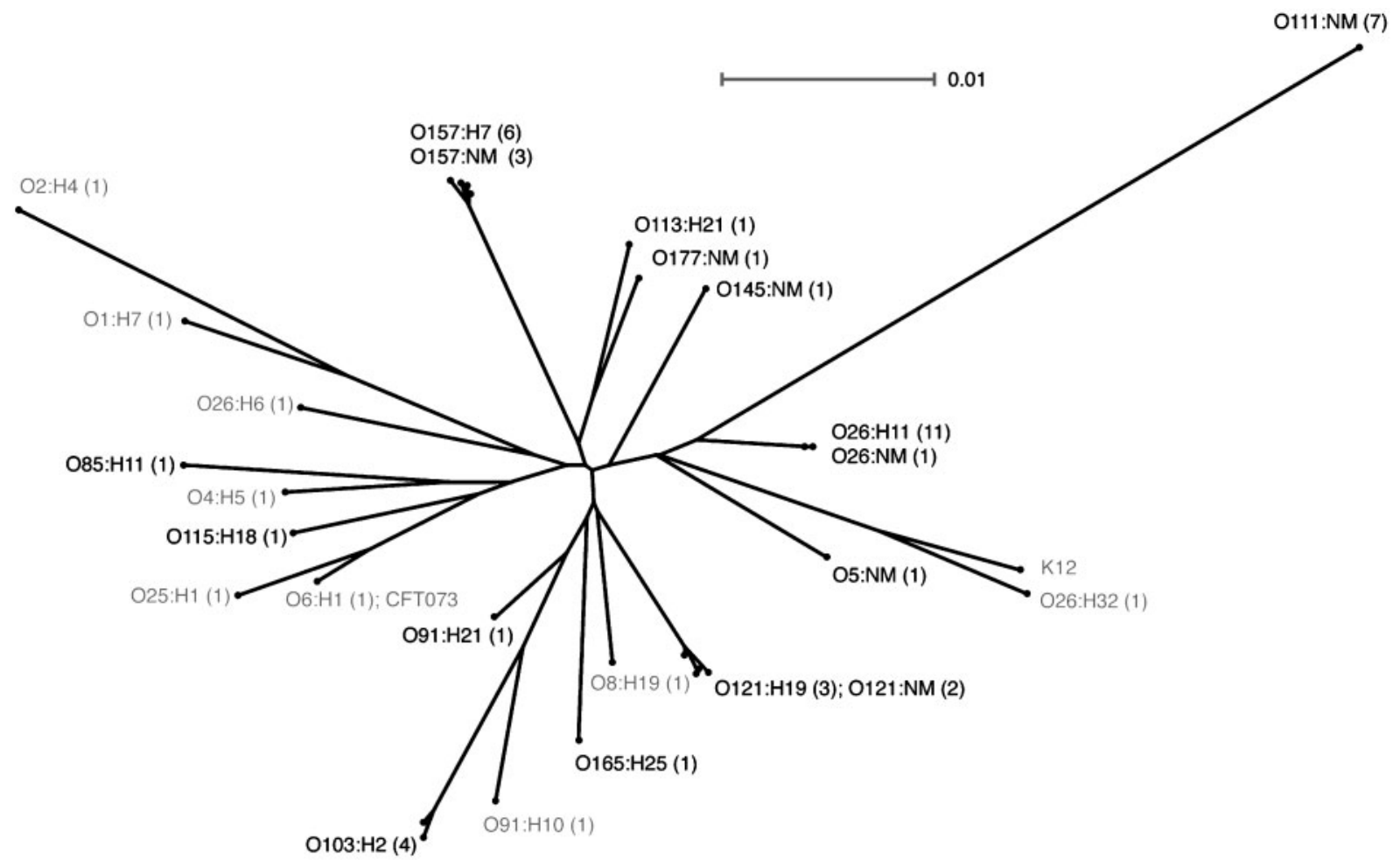

Fig. 2. Phylogeny of the concatenated segments of $g n d, g c l, p p k$ and relA encoded by $E$. coli. This is based upon a neighbourjoining tree constructed with Hasegawa-Kishino-Yano (HKY85) distance correction. Sequences obtained from GenBank are identified in Methods. Shiga toxin-producing serotypes are indicated in black type, and strains not encoding stx are indicated in grey. The number of sequences per serotype is indicated in parentheses. Bar, scale of the distance score.

single nucleotide polymorphism compared to the other O157 strains, but otherwise the gnd alleles were conserved within STEC serogroup classifications. Furthermore, nontoxin encoding strains of O157, O26, O55, O6 and O117 encoded distinct gnd alleles compared to STEC strains of the same serogroup. Sequence typing of gnd was, therefore, a promising molecular method correlating minimally with the O-serogroup of clinical STEC strains. The O111:NM STEC and non-toxin-producing O55 strains encoded gnd sequences outlying from the main cluster (Fig. 3 ) and these were homologous to Citrobacter spp. gnd alleles (Nelson \& Selander, 1994). However, since pure bacterial isolates are preferred for preparation of DNA sequencing template, all isolates undergoing gnd DNA sequence-based serogrouping should previously be classified as STEC.

During the course of this study, outbreak-related isolates of non-O157 STEC were sent to the National Microbiology Laboratory for serotyping and genetic characterization. The gnd sequence data for each of isolates $05-6541$ to $05-6543$ clustered with known O121 strains (Fig. 3). A concurrent non-O157 sporadic isolate (05-6544) was also examined at gnd and this sequence clustered with known O26:H11 strains (Fig. 3). Strain 06-5121 was isolated from a hospitalized patient with haemolytic uraemic syndrome and the gnd sequence of this strain was $99.8 \%$ identical to a known O177 : NM isolate (Fig. 3). In correlation with these molecular data, subsequent serotyping using traditional methodologies characterized these isolates as $\mathrm{O} 121: \mathrm{H} 19$, $\mathrm{O} 26: \mathrm{H} 11$ and O177:NM. The gnd DNA sequence-based serogrouping method therefore provided an advantageous alternative to $\mathrm{O}$-specific immunoreagents during these crises. Over 55 serogroups of STEC have been reported to be associated with human disease (Johnson et al., 2006), and an international panel of STEC strains from each serogroup, including the emerging sorbitol-fermenting O157, will be required to further validate this method.

The proportion of synonymous and nonsynonymous mutations was calculated for each locus from the accumulated DNA sequence data (Table 3). As expected for core loci, the majority of mutations were synonymous (dN/ dS $<1$ ), but the gnd locus had the greatest number of nonsynonymous sites. This locus has already been identified as a polymorphic E. coli locus compared to other core loci (Bisercic et al., 1991; Nelson \& Selander, 1994; Dykhuizen \& Green, 1991). A comparable ratio of synonymous versus nonsynonymous mutations was also reported by Bisercic et al. (1991). Genetic diversity at gnd arose in parallel to the extensive diversity and recombination that occurred at the neighbouring $\mathrm{O}$-antigen gene cluster, and it is likely that these two genetic traits were 


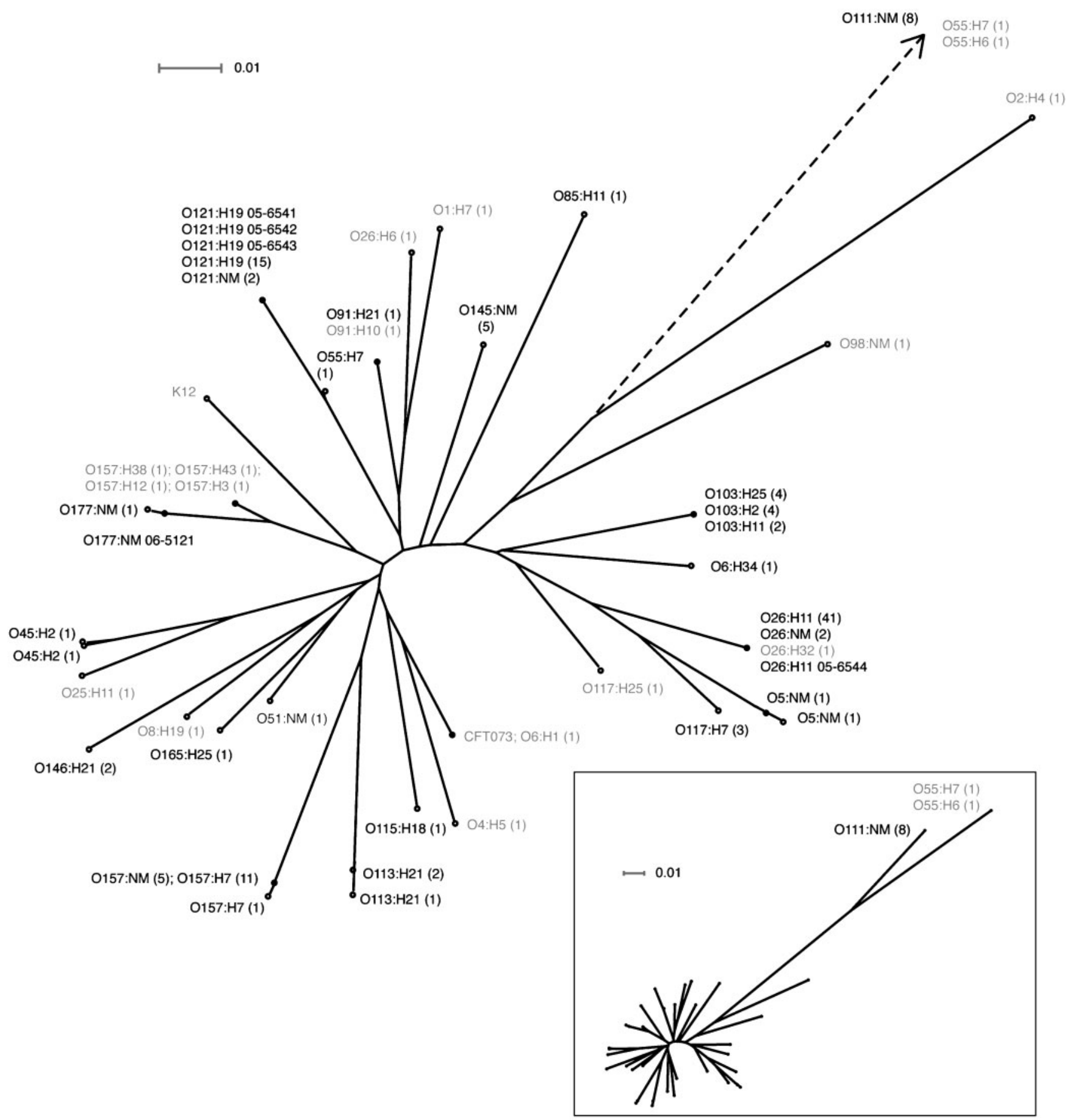

Fig. 3. Phylogeny of the gnd locus encoded by E. coli. This is based upon a neighbour-joining tree constructed with Hasegawa-Kishino-Yano (HKY85) distance correction. Sequences obtained from GenBank are identified in Methods. Shiga toxin-producing serotypes are indicated in black type, and strains not encoding stx are indicated in grey. The number of sequences per serotype is indicated in parentheses. Strain identification numbers are indicated for outbreak-associated clinical isolates. The dotted line indicates outlying gnd sequences, which are presented in relation to the entire dataset in the inset. Bar, scale of the distance score. 
Table 3. Genetic diversity of the protein-encoding loci of $E$. coli sequenced in this study

For comparative purposes, multiple statistics for the gnd locus are presented as increasing numbers of serotypes and strains were analysed.

\begin{tabular}{|c|c|c|c|c|c|c|c|}
\hline Target & $\begin{array}{c}\text { No. of } \\
\text { sequences }^{*}\end{array}$ & $\begin{array}{c}\text { No. of } \\
\text { serotypes } \dagger\end{array}$ & $\begin{array}{l}\text { Size of target } \\
\text { (bp) }\end{array}$ & $\begin{array}{l}\text { No. of polymorphic } \\
\text { sites }(\pi) \ddagger\end{array}$ & $\begin{array}{l}\text { No. of synonymous } \\
\text { polymorphic sites }\end{array}$ & $\begin{array}{l}\text { No. of nonsynonymous } \\
\text { polymorphic sites }\end{array}$ & $\mathrm{dN} / \mathrm{dS} \S$ \\
\hline \multirow[t]{2}{*}{ gnd } & 47 & 42 & 643 & $210(0.067)$ & 189 & 21 & 0.035 \\
\hline & 17 & 16 & 643 & $173(0.062)$ & 154 & 19 & 0.030 \\
\hline$g c l$ & 26 & 26 & 654 & $68(0.023)$ & 61 & 7 & 0.023 \\
\hline relA & 30 & 26 & 425 & $42(0.019)$ & 41 & 1 & 0.002 \\
\hline$f t s Z$ & 16 & 16 & 404 & $17(0.010)$ & 17 & 0 & 0.000 \\
\hline metA & 16 & 16 & 559 & $36(0.015)$ & 29 & 7 & 0.115 \\
\hline$m e t G$ & 16 & 16 & 434 & $46(0.024)$ & 42 & 4 & 0.021 \\
\hline$p p k$ & 28 & 26 & 701 & $40(0.013)$ & 39 & 1 & 0.005 \\
\hline
\end{tabular}

${ }^{\star}$ Identical DNA sequences belonging to the same $\mathrm{O}: \mathrm{H}$ serotype were included only once.

$\dagger$ Minimally includes the serotypes indicated in Fig. 1 (when no. of serotypes=16), in Fig. 2. (when no. of serotypes=26) or in Fig. 3 (when no. of serotypes $=42$ ).

$\ddagger \pi$, Measure of genetic diversity.

§Rate of nonsynonymous and synonymous mutations.

co-inherited between lineages (Tarr et al., 2000; Nelson \& Selander, 1994). To our knowledge, there is no indication that O-serogroups that encode similar gnd alleles (e.g. STEC O121 and O55) also encode similar O-antigen gene clusters, nor are the antigens themselves similar. The potential utility of a locus subject to recombination between genera might be seemingly limited for the purpose of molecular-based serogrouping; however, we currently observed conserved STEC serogroup-specific genetic polymorphisms at the gnd locus. Between strains of an individual STEC O-serogroup we observed conserved gnd alleles, and no serogroup encoded a gnd allele that was identical to another serogroup. This study provides a simple method for molecular-based serogrouping of E. coli strains encoding stx, which can be detected by a wealth of molecular reagents (Gilmour et al., 2006; Hsu et al., 2005; Nielsen \& Andersen, 2003; Reischl et al., 2002; Wang et al., 2002). This method was used to characterize O121: H19, $\mathrm{O} 26: \mathrm{H} 11$ and $\mathrm{O} 177: \mathrm{NM}$ clinical isolates prior to serological confirmation during an outbreak investigation, and could, therefore, improve the scope of STEC molecular diagnostics beyond the $\mathrm{O} 157$ serogroup.

\section{ACKNOWLEDGEMENTS}

We thank Tim Mailman and Nova Scotia Public Health for providing outbreak-associated strains, and John Wylie at the Manitoba Cadham Provincial Laboratory, Winnipeg, Manitoba, Canada, Ana Paccagnella at the British Columbia Centre for Disease Control, Vancouver, British Columbia, Canada, and Yvonne Yaschuk at New Brunswick Public Health, Saint John, New Brunswick, Canada, for providing strains. We also thank Julie Walsh, Helen Tabor, Dobryan Tracz and Clifford Clark for helpful discussions. Oligonucleotide synthesis and DNA sequencing was performed by the DNA core facility, and serology was performed by the Serotyping and Identification Unit at the National Microbiology Laboratory. This work was supported by the Office of Biotechnology and Science.

\section{REFERENCES}

Adiri, R. S., Gophna, U. \& Ron, E. Z. (2003). Multilocus sequence typing (MLST) of Escherichia coli O78 strains. FEMS Microbiol Lett 222, 199-203.

Beutin, L., Kaulfuss, S., Herold, S., Oswald, E. \& Schmidt, H. (2005). Genetic analysis of enteropathogenic and enterohemorrhagic Escherichia coli serogroup $\mathrm{O} 103$ strains by molecular typing of virulence and housekeeping genes and pulsed-field gel electrophoresis. J Clin Microbiol 43, 1552-1563.

Bisercic, M., Feutrier, J. Y. \& Reeves, P. R. (1991). Nucleotide sequences of the gnd genes from nine natural isolates of Escherichia coli: evidence of intragenic recombination as a contributing factor in the evolution of the polymorphic gnd locus. J Bacteriol 173, 3894-3900.

Coimbra, R. S., Grimont, F., Lenormand, P., Burguiere, P., Beutin, L. \& Grimont, P. A. (2000). Identification of Escherichia coli Oserogroups by restriction of the amplified O-antigen gene cluster ( $r f b$-RFLP). Res Microbiol 151, 639-654.

DebRoy, C., Roberts, E., Kundrat, J., Davis, M. A., Briggs, C. E. \& Fratamico, P. M. (2004). Detection of Escherichia coli serogroups O26 and $\mathrm{O} 113$ by PCR amplification of the $w z x$ and $w z y$ genes. Appl Environ Microbiol 70, 1830-1832.

D'Souza, J. M., Wang, L. \& Reeves, P. (2002). Sequence of the Escherichia coli $\mathrm{O} 26 \mathrm{O}$ antigen gene cluster and identification of $\mathrm{O} 26$ specific genes. Gene 297, 123-127.

DebRoy, C., Fratamico, P. M., Roberts, E., Davis, M. A. \& Liu, Y. (2005). Development of PCR assays targeting genes in O-antigen gene clusters for detection and identification of Escherichia coli $\mathrm{O} 45$ and O55 serogroups. Appl Environ Microbiol 71, 4919-4924.

Dykhuizen, D. E. \& Green, L. (1991). Recombination in Escherichia coli and the definition of biological species. J Bacteriol 173, 7257-7268. 
Ewing, W. H. (1986). The genus Escherichia. In Edwards \& Ewing's Identification of Enterobacteriaceae, 4th edn, pp. 93-134. Edited by P. R. Edwards and W. H. Ewing. New York: Elsevier.

Fey, P. D., Wickert, R. S., Rupp, M. E., Safranek, T. J. \& Hinrichs, S. H. (2000). Prevalence of non-O157:H7 shiga toxin-producing Escherichia coli in diarrheal stool samples from Nebraska. Emerg Infect Dis 6, 530-533.

Fratamico, P. M., DebRoy, C., Strobaugh, T. P., Jr \& Chen, C. Y. (2005). DNA sequence of the Escherichia coli O103 O antigen gene cluster and detection of enterohemorrhagic E. coli O103 by PCR amplification of the wzx and wzy genes. Can J Microbiol 51, 515-522.

Gilmour, M. W., Cote, T., Munro, J., Chui, L., Wylie, J., Isaac-Renton, J., Horsman, G., Tracz, D. M., Andrysiak, A. \& Ng, L. K. (2005). Multilocus sequence typing of Escherichia coli O26:H11 isolates carrying stx in Canada does not identify genetic diversity. J Clin Microbiol 43, 5319-5323.

Gilmour, M. W., Tracz, D. M., Andrysiak, A. K., Clark, C. G., Tyson, S., Severini, A. \& Ng, L. K. (2006). Use of the espZ gene encoded in the locus of enterocyte effacement for molecular typing of Shiga toxinproducing Escherichia coli. J Clin Microbiol 44, 449-458.

Girardeau, J. P., Dalmasso, A., Bertin, Y., Ducrot, C., Bord, S., Livrelli, V., Vernozy-Rozand, C. \& Martin, C. (2005). Association of virulence genotype with phylogenetic background in comparison to different seropathotypes of Shiga toxin-producing Escherichia coli isolates. $J$ Clin Microbiol 43, 6098-6107.

Hsu, C. F., Tsai, T. Y. \& Pan, T. M. (2005). Use of the duplex TaqMan PCR system for detection of Shiga-like toxin-producing Escherichia coli O157. J Clin Microbiol 43, 2668-2673.

Huson, D. H. (1998). SplitsTree: analyzing and visualizing evolutionary data. Bioinformatics 14, 68-73.

Jelacic, J. K., Damrow, T., Chen, G. S., Jelacic, S., Bielaszewska, M., Ciol, M., Carvalho, H. M., Melton-Celsa, A. R., O’Brien, A. D. \& Tarr, P. I. (2003). Shiga toxin-producing Escherichia coli in Montana: bacterial genotypes and clinical profiles. J Infect Dis 188, 719-729.

Johnson, K. E., Thorpe, C. M. \& Sears, C. L. (2006). The emerging clinical importance of non-O157 Shiga toxin-producing Escherichia coli. Clin Infect Dis 43, 1587-1596.

Karch, H., Tarr, P. I. \& Bielaszewska, M. (2005). Enterohaemorrhagic Escherichia coli in human medicine. Int J Med Microbiol 295, 405-418.

Karmali, M. A., Mascarenhas, M., Shen, S., Ziebell, K., Johnson, S., Reid-Smith, R., Isaac-Renton, J., Clark, C., Rahn, K. \& Kaper, J. B. (2003). Association of genomic O island 122 of Escherichia coli EDL 933 with verocytotoxin-producing Escherichia coli seropathotypes that are linked to epidemic and/or serious disease. J Clin Microbiol 41, 4930-4940.

Liu, Y. \& Fratamico, P. (2006). Escherichia coli $\mathrm{O}$ antigen typing using DNA microarrays. Mol Cell Probes 20, 239-244.

Nelson, K. \& Selander, R. K. (1994). Intergeneric transfer and recombination of the 6-phosphogluconate dehydrogenase gene ( $g n d$ ) in enteric bacteria. Proc Natl Acad Sci U S A 91, 10227-10231.

Nielsen, E. M. \& Andersen, M. T. (2003). Detection and characterization of verocytotoxin-producing Escherichia coli by automated 5' nuclease PCR assay. J Clin Microbiol 41, 2884-2893.
Noller, A. C., McEllistrem, M. C., Stine, O. C., Morris, J. G., Jr, Boxrud, D. J., Dixon, B. \& Harrison, L. H. (2003). Multilocus sequence typing reveals a lack of diversity among Escherichia coli O157:H7 isolates that are distinct by pulsed-field gel electrophoresis. J Clin Microbiol 41, 675-679.

Paton, A. W. \& Paton, J. C. (1999a). Direct detection of Shiga toxigenic Escherichia coli strains belonging to serogroups O111, O157, and O113 by multiplex PCR. J Clin Microbiol 37, 3362-3365.

Paton, A. W. \& Paton, J. C. (1999b). Molecular characterization of the locus encoding biosynthesis of the lipopolysaccharide $\mathrm{O}$ antigen of Escherichia coli serotype O113. Infect Immun 67, 5930-5937.

Paton, A. W., Ratcliff, R. M., Doyle, R. M., Seymour-Murray, J., Davos, D., Lanser, J. A. \& Paton, J. C. (1996). Molecular microbiological investigation of an outbreak of hemolytic-uremic syndrome caused by dry fermented sausage contaminated with Shigalike toxin-producing Escherichia coli. J Clin Microbiol 34, 1622-1627.

Perelle, S., Dilasser, F., Grout, J. \& Fach, P. (2005). Detection of Escherichia coli serogroup $\mathrm{O} 103$ by real-time polymerase chain reaction. J Appl Microbiol 98, 1162-1168.

Prager, R., Annemuller, S. \& Tschape, H. (2005). Diversity of virulence patterns among Shiga toxin-producing Escherichia coli from human clinical cases - need for more detailed diagnostics. Int J Med Microbiol 295, 29-38.

Reischl, U., Youssef, M. T., Kilwinski, J., Lehn, N., Zhang, W. L., Karch, H. \& Strockbine, N. A. (2002). Real-time fluorescence PCR assays for detection and characterization of Shiga toxin, intimin, and enterohemolysin genes from Shiga toxin-producing Escherichia coli. J Clin Microbiol 40, 2555-2565.

Rozas, J., Sanchez-DelBarrio, J. C., Messeguer, X. \& Rozas, R. (2003). DnaSP, DNA polymorphism analyses by the coalescent and other methods. Bioinformatics 19, 2496-2497.

Tarr, P. I., Schoening, L. M., Yea, Y. L., Ward, T. R., Jelacic, S. \& Whittam, T. S. (2000). Acquisition of the $r f b$-gnd cluster in evolution of Escherichia coli O55 and O157. J Bacteriol 182, 6183-6191.

Tarr, C. L., Large, T. M., Moeller, C. L., Lacher, D. W., Tarr, P. I., Acheson, D. W. \& Whittam, T. S. (2002). Molecular characterization of a serotype O121: H19 clone, a distinct Shiga toxin-producing clone of pathogenic Escherichia coli. Infect Immun 70, 6853-6859.

Thompson, L. H., Giercke, S., Beaudoin, C., Woodward, D. L. \& Wylie, J. L. (2005). Enhanced surveillance of non-O157 verotoxin-producing Escherichia coli in human stool samples from Manitoba. Can J Infect Dis Med Microbiol 16, 329-334.

Wang, L., Curd, H., Qu, W. \& Reeves, P. R. (1998). Sequencing of Escherichia coli $\mathrm{O} 111 \mathrm{O}$-antigen gene cluster and identification of O111-specific genes. J Clin Microbiol 36, 3182-3187.

Wang, G., Clark, C. G. \& Rodgers, F. G. (2002). Detection in Escherichia coli of the genes encoding the major virulence factors, the genes defining the $\mathrm{O} 157: \mathrm{H} 7$ serotype, and components of the type 2 Shiga toxin family by multiplex PCR. J Clin Microbiol 40, 3613-3619.

Woodward, D. L., Clark, C. G., Caldeira, R. A., Ahmed, R. \& Rodgers, F. G. (2002). Verotoxigenic Escherichia coli (VTEC): a major public health threat in Canada. Can J Infect Dis 13, 321-330. 